\title{
A model for the reversal of the toroidal rotation in tokamak
}

\author{
Florin Spineanu and Madalina Vlad \\ National Institute of Laser, Plasma and Radiation Physics \\ Bucharest 077125, Romania
}

\begin{abstract}
The transition from toroidal counter- to co- rotation in the core plasma has been observed at $\mathrm{L}$ to $\mathrm{H}$ transition in several tokamaks. Spontaneous reversal has also been observed in TCV beyond a threshold in the density. We develop a model based on the following phenomenology: (1) the increase of the gradient of the pressure triggers formation on a fast time scale of cells of convection (similar to Rayleigh-Benard (RB), but with a single sign of vorticity); (2) poloidal rotation is induced by the envelope of the peripheric velocity of the convection cells; via the baroclinic term the gradients of temperature and density sustain the poloidal rotation against the decay due to the parallel viscosity; (3) the fast increase of poloidal flow induces a high time derivative of the radial electric field; (4) the neoclassical polarization creates a series of parallel accelerations (kiks on each bounce) of the trapped ions, leading to an increase of the toroidal precession or to its reversal; the source of energy is the work done by the radial electric field. (5) the diffusion transfers on resistive scale the toroidal momentum from the trapped ions to the untrapped ones. The correlated interactions are examined and the estimated time scales are found to be compatible with the observations.
\end{abstract}

\section{Introduction}

The spontaneous reversal of the direction of the toroidal rotation in tokamak has been observed as a change accompanying the $\mathrm{L}$ to $\mathrm{H}$ transition or a slow variation of a plasma parameter, like the current or the magnetic field [1], [2], [3], [4], [5]. Spontaneous reversal has also been observed in TCV beyond 
a threshold in the density [6]. A review of these experimental facts can be found in Refs. [7] and [8].

There are several properties of the state of the system that seem to be correlated with the transition consisting of the flow reversal. There is a strong radial gradient of temperature which is sustained by external input of energy into plasma. In general fluid configurations placed under strong thermal stress [for example in the classical Rayleigh-Benard (RB) system] the purely conductive state is replaced by a convective regime through generation of cells of convection [9]. The transport of heat and of momentum and angular momentum (via the Reynolds stress) become much more efficient compared with the diffusive regime. The onset of the convection structure takes place on short time scale compared with conduction or flow time scales. The event leading to formation of a regular convection pattern starts with the generation of isolated hot and cold streamers (also called "plumes" or "thermals") that advance into the fluid environment [10. The head of the streamer is deformed through interaction with the static fluid leading to "mushroom" shapes also known from Rayleigh-Taylor experiments. The streamer breaks down leaving vortices of two signs. These vortices are merging eventually creating the large scale flow, a mechanism which is universally associated to relaxation [11], [12. Under strong temperature gradient the whole process is very fast.

The presence and/or the possible generation of closed, quasi-coherent convective flows in the meridional cross section of the tokamak plasma is a well investigated subject. The particular case of the zonal flows is included as a subset in this wide class of organized flows (for reviews see [13], 14], [15]). Various mechanisms have been identified to be able to transfer energy from the drift wave turbulence to the flow in a convective structure extending over a substantial part of the plasma cross section. Modulational instability, wave-kinetic dynamical transfers in the spectral space, multi-scale interactions leading to negative viscosity are usually invoked [16], [17], [18], [19]. A unifying and universal principle is the inverse cascade of energy in the two-dimensional turbulence [11, [20], 21], 22], 23]. Vortical structures consisting of robust and coherent flow have been identified in regimes governed by the Hasegawa-Mima and Hasegawa-Wakatani equations [15]. Due to the essential role of the vectorial nonlinearity the space scales of the these structures is initially at the level of the ion Larmor radius. The processes or merging lead further to creation of large scale structure. Another mechanism that has been studied is the tilting instability of eddies with creation, via Reynolds stress, of flows over large spatial regions [24]. The structure is usually assumed with alternate sense of rotation, for several reasons. One of 
them is the conservation of the angular momentum integrated over the space occupied by the cells of convection. This conditions is however imposed to closed systems, and even for them is not quite stringent [25]. A different reason comes from assuming the flow as a perturbation, expanding in Fourier series and retaining the lowest order which by its nature is periodic and leads to vortices with alternating sense of rotation [9]. It also provides compatible flows at the common boundaries of the neighbor cells. The latter argument should not be seen as a strong restriction if the distance between the rolls of convection is sufficiently large. Finally we mention a condition related with the space-time invariances of the equations, which appears to underlay this choice of the pattern of flow. Taking as a propotype the Hasegawa-Mima equation one notes the invariance to reversal of the sign of the vorticity, which implicitely imposes the presence in the solution of vortices of both signs. However it has been shown that besides the vectorial nonlinearity the scalar or Korteweg-deVries nonlinearity, of the type $\psi(\partial \psi / \partial y)$ where $\psi$ is the streamfunction and $y$ the poloidal coordinate, is likely to be present in the equation [26], [27]. Although it does not influence the smaller scales where the instabilities are developing, it controls the large scales due to the lower differential degree. This breaks the invariance and allows solutions consisting of a set of rolls with unique sense of rotation. We argue that rolls of convection having the same direction of rotating flow are not excluded by any characteristic of the differential equations governing the quasi-twodimensional tokamak plasma and are even more efficient for the transport of angular momentum in the meridional section. This pattern of flow has been derived [28] and it arises in many similar situations. In the science of atmosphere it is known that a distribution of azimuthal velocity in a vortex can be affected by perturbations leading to secondary vortices that move around the core of the parent system [29]. The geometry of the flow takes the form of a polygonal eye-wall, which means that a set of smaller vortices are placed symmetrically, with their centers placed on a circle around the eye-wall axis [30]. They all have the same sign of vorticity. Other examples are accretion disks [31] and the Ranque-Hilsch vortex tube [32].

In tokamak there is a poloidal rotation at equilibrium, due to the neoclassical processes. The poloidal flow is sheared since part of the plasma cross section is in plateau - banana and part is in Pfirsch-Schluter regime [33]. This just constitutes a seed which may favor a choice of the direction of rotation of the rolls that are going to be created.

A source of flow that appears to be less investigated is the baroclinic term arising in the momentum equation of the ion component when it is transformed by a rotational operator into the equation for toroidally projected vorticity. It is well suited to exhibit local velocity shear that may accelerate 
plasma flow, leading for example to generation of streamers. As shown below, the streamers constitute a basic agent for building up larger scale flows.

It is well known that the RB transition from purely conductive to a convective regime takes place at a threshold, where the buoyancy force overcomes the viscosity [9]. In the present case the threshold has a different nature. The drive force in the early stage is the baroclinic term, dependent on the main gradients of the density and temperature, which are supported by the external input. Convective cells are likely to be created, as shown by numerous investigations (analytical, numerical, observations). The pattern should obey the symmetries required by the basic neoclassical rotation which favors formation of rolls all rotating in the same sense, equivalently, with the same sign of toroidally-directed vorticity. There is no restriction upon this choice coming from the conservation of the angular momentum in the meridional plane since momentum is carried away to the boundary (the system is open). Now, the peripheric velocities of the chain of large scale vortical cells constitues an effective poloidal rotation. The magnetic pumping opposes the poloidal rotation via the parallel viscosity which acts on a time scale determined by the ion-ion collision frequency [34]. This leads us to formulate a threshold condition: the gradients of density and temperature should be sufficiently high such as the baroclinic term to sustain the rolls and their induced poloidal flow against the parallel viscosity damping. The detailed examination of this threshold condition requires more quantitative analysis and will not be of the present concern.

We develop a model based on the following phenomenology:

(1) the increase of the gradient of the pressure triggers formation of cells of convection. The process is similar to the transition from purely conductive to convective regime in Rayleigh-Benard fluid but the rolls of convection have a single sign of vorticity; the baroclinic term drives a fast onset of this pattern of flow;

(2) the peripheric velocities of the convection cells (with unique sense of rotation) coalesce into an envelope which is equivalent to a poloidal rotation; in usual circumstances the poloidal rotation is damped by the parallel viscosity (magnetic pumping) but this is now overcomed by the Reynolds stress in the regular pattern which is directly sustained by the gradients of density and temperature. These are, in turn, sustained by the external input.

(3) the fast increase of poloidal flow induces a high time derivative of the radial electric field $E_{r}$. The radial momentum balance would allow to divide the effect of an increase of the $v_{\theta}$ term to the two other terms, containing the diamagnetic and respectively the toroidal velocities. However the reaction of the diamagnetic velocity is slower since it depends on the change of the 
density profile and the change of the toroidal flow velocity is mediated by a neoclassical effect.

(4) the neoclassical polarization induced by the variation of the radial electric field creates a series of parallel accelerations (kick on every bounce) of the trapped ions, leading to an increase of the toroidal precession or to its reversal; the source of energy is the work done by the fast transient growth of $E_{r}$ supported in turn by the pressure gradient through the convection.

(5) the diffusion transfers on resistive scale the toroidal momentum from the trapped ions to the untrapped ones, inducing the reversal of direction.

The point (4) roughly describes this situation: on half of the banana (say in the same direction as the magnetic field $\mathbf{B}$ ) the trapped ion feels a certain radial electric field and when it returns on the other half of the banana orbit (opposite to B) it already is acted upon by a different radial electric field. The key is that the change of the radial electric field and the ion bounce on banana take place on comparable time scales. This is possible due to the fast onset of convection and can be the explanation of the fast transitions observed in experiments [35].

Our model is similar to an "inverse Ranque-Hilsch" effect. In the RanqueHilsch vortex (not fully understood at this time) a strong rotation of a gas leads to temperature separation [36] and it is supposed that coherent vortical structures that breaks the azimuthal symmetry of the flow are responsible for the creation of the gradient of temperature [32]. In our case we dispose of the strong temperature gradient across the plasma section and the onset of cells of convection (which break the azimuthal symmetry) would create the other element, i.e. the poloidal flow. The process is sustained by the heat and density input which is converted into mechanical stress (radial variation of poloidal rotation) by the cells of convection. It is transitory with a fast phase (when the reversal occurs) followed by a slower variation which keeps the time derivative of the radial electric field at the level required for balancing the parallel viscosity damping.

We focus in the present work on the logical consistency of the argument, examining the contributing physical processes under certain simplifications. The overall result confirms the validity of the model and justifies further study.

The paper is organised as follows: we explain the role of the baroclinic term in sustaining the perturbations consisting of hot streamers advancing along the minor radius. The estimations shows that the time scale of the convection is very fast. Separately we provide an argument for the poloidal flow of the density toward the place of origin of the streamer, preventing a local depression of density and suppression or breaking up of the hot streamer. This argument is based on another manifestation of the baroclinic effect, 
which in this case reveals the possiblity of the instability called Chaplygin gas, or anomalous polytropic. The next section is devoted to demonstrate that the peripheric velocity of the rolls of convection leads to an effective poloidal flow. The argument is here geometric and on this basis one can easily superpose physical processes like Reynolds stress and collisional diffusion of the momentum in the vecinity of the rolls of convection. The last part consists of simply adapting the arguments of Hinton and Robertson [37] to show that the neoclassical polarization induces an acceleration of the toroidal motion of the bananas.

\section{The hot streamers in the early phase of the onset of the convection rolls}

We consider a perturbation in the two-dimensional section of a tokamak plasma, consisting of the radial displacement of an element of plasma from the radius $r_{0}$ to higher radii, i.e. to toward the edge. For fast motion the element preserves its temperature and advances as a stream of hot plasma to higher $r$. The Figure 1 shows the geometry of the perturbation fixing also the system of reference $(x \equiv r, y \equiv r \theta, z)$. The smooth surface represents the equilibrium pressure profile on which it is superposed the perturbation related to the advancing hot streamer. The curved arrow shows the effect of the baroclinic term. The two arrows at the right of the perturbations have the following meaning: the one directed against the perturbation represents the $\boldsymbol{\nabla} T$ of the hot stream relative to the environment. The one poining approximately in the inverse $x \equiv r$ direction is the gradient of the equilibrium density (or mass), $\nabla \rho$.

The density at the radius where the streamer begins its motion is $n\left(r_{0}\right)$ and the temperature at the same location is $T\left(r_{0}\right)$. The displacement of the hot plasma element along the radius is $\delta r=r-r_{0}$ which is positive in our system. On this distance the equilibrium (background) pressure has varied and has the value

$$
p(r)=p\left(r_{0}\right)+\frac{d p}{d r} \delta r=p\left(r_{0}\right)-p(r) \frac{1}{\left|L_{p}\right|} \delta r
$$

where $L_{p}<0$ on an usual equilibrium pressure profile. This $p(r)$ is the pressure in the environment plasma at a distance $\delta r$ from the point of initialization of the thermal streamer. Assuming that there is no loss of heat via diffusion the hot stream will have at the same position the pressure $p\left(r_{0}\right)$. The gradient due to this contrast between the hot streamer and the environment is directed (along poloidal direction at $r$ ) from the boundary of the 
thermal streamer to its axis. We will choose to look at the left side of the hot streamer which means that the gradient of the pressure is directed along the positive $y$ axis, $\widehat{\mathbf{e}}_{\theta}$. The width of the hot stream is of the order of the radius of the roll, $R$ and the gradient of pressure transversal on the axis of the streamer is

$$
\frac{\delta p}{R}=\eta \frac{p\left(r_{0}\right)-p(r)}{R}=\eta p(r) \frac{1}{\left|L_{p}\right|} \delta r \frac{1}{R}
$$

In order to keep a realistic set of assumptions we must consider the loss of heat from the streamer via conduction to the environment plasma. An elementary option is to introduce an arbitrary nondimensional small factor $\eta$ and consider from now on the fraction $\eta$ of the perturbation of the pressure. Now, the gradient of the perturbed pressure interacts with the equilibrium gradient of the density $\rho_{0}$ to give the baroclinic term

$$
\begin{aligned}
\frac{1}{\rho_{0}^{2}} \nabla \rho_{0} \times \nabla p & =\frac{1}{\rho_{0}^{2}}\left|\nabla \rho_{0}\right|\left(-\widehat{\mathbf{e}}_{r}\right) \times|\nabla p|\left(\widehat{\mathbf{e}}_{\theta}\right) \\
& =\frac{1}{\rho_{0}^{2}}\left|\nabla \rho_{0}\right||\nabla p|\left(-\widehat{\mathbf{e}}_{z}\right)
\end{aligned}
$$

and using Eq.(2) we have

$$
\frac{1}{\rho_{0}^{2}} \nabla \rho_{0} \times \nabla p=\frac{1}{m_{i} n(r)} \frac{1}{\left|L_{n}\right|} \eta \frac{p(r)}{\left|L_{p}\right|} \frac{\delta r}{R}\left(-\widehat{\mathbf{e}}_{z}\right)
$$

The two quantities: the depth of penetration of the streamer $\delta r$ and the radius of the cell of convection $R$ produced by the roll-up of the streamer have similar magnitudes and we approximate

$$
\frac{\delta r}{R} \sim 1
$$

The baroclinic term is directed along the negative $z$ (toroidal) axis and enhances the local gradients of the velocity of the streamer: the velocity on the axis of the hot streamer is accelerated. With this specification of the direction of the enhancement of the velocity by the time-derivative of the local vorticity, we will only take from now on its absolute value

$$
\left|\frac{\partial \omega}{\partial t}\right| \sim \frac{1}{\delta t} \frac{v}{R} \sim \frac{1}{m_{i} n(r)} \frac{1}{\left|L_{n}\right|} \eta \frac{p(r)}{\left|L_{p}\right|}
$$

Here $v \equiv v_{r}$ is the speed of the hot streamer, along its axis. The interval of time $\delta t$ will later be taken a characteristic turn-over of the convection roll. The gradient length of the equilibrium pressure is

$$
\frac{1}{L_{p}}=\frac{1}{p} \frac{\partial p}{\partial r}=\frac{1}{n T}\left(T \frac{\partial n}{\partial r}+n \frac{\partial T}{\partial r}\right)=\frac{1}{L_{n}}+\frac{n}{p} \frac{\partial T}{\partial r}
$$


with both terms having the same sign, negative along the increasing minor radius direction. From Eqs.(6) and (7) we have the estimation

$$
\frac{1}{\delta t} \frac{v}{R} \sim \eta \frac{T}{m_{i}}\left(\frac{1}{\left|L_{n}\right|^{2}}+\frac{1}{\left|L_{n}\right|} \frac{1}{\left|L_{T}\right|}\right)
$$

Now, if we assume that the gradient length of the density is much larger than $\left|L_{T}\right|$, we will retain only the second term in the paranthesis

$$
\frac{1}{\delta t} \frac{v}{R} \sim \eta \frac{1}{m_{i}} \frac{1}{\left|L_{n}\right|}|\nabla T|
$$

Taking

$$
\delta t \sim \frac{R}{v}
$$

we obtain

$$
v^{2} \sim R^{2} \eta \frac{1}{m_{i}\left|L_{n}\right|}|\nabla T|
$$

The gradient of the equilibrium temperature at the position and on the spatial extension of the convection roll must be a fraction of the gross estimation $T / R$ and we introduce an arbitrary nondimansional small parameter $\lambda$

$$
|\nabla T| \sim \lambda \frac{T}{R}
$$

it results

$$
v^{2} \sim R^{2} \eta \lambda \frac{1}{m_{i}\left|L_{n}\right|} \frac{T}{R}=\eta \lambda \frac{R}{\left|L_{n}\right|} v_{i, t h}^{2}
$$

Except for the two small parameters $\eta$ and $\lambda$ this formula shows that the speed of rotation at the periphery of the roll is very high, comparable with the ion thermal velocity. Including a rough estimation for the conductive loss (similar to the Rayleigh-Benard case) by the factor $\eta=0.1$ and the variation of the equilibrium temperature over the cell's extension, by the factor $\lambda=0.1$, we still get a high value $v \sim 0.1 \times v_{t h, i}$.

\section{The local perturbation of the density along the poloidal direction}

\subsection{The instability of the Chaplygin-type or negative pressure}

The perturbation is initiated at a radius $r_{0}$, which means that the density in a small region around the circle $r_{0}$ will flow toward the point (say $\theta_{0}$ ) where 
the streamer originates. There the poloidal aflux is converted into radial flow of the hot streamer. This radial flow is accelerated by the baroclinic effect and from the Eq.(13) we see that the speed can reach high values. A local depression of density can occur if the rate of filling with density the foot of the streamer takes place at a rate which is slower than that with which it is removed by the streamer. This is an important aspect which we examine in the following. The continuity equation with only the velocity along the streamer is

$$
\frac{\partial n}{\partial r}+\frac{\partial}{\partial r}\left(v_{r} n\right)=0
$$

or

$$
\frac{\partial}{\partial t} \ln n+\frac{\partial v_{r}}{\partial r}+v_{r} \frac{\partial}{\partial r} \ln n=0
$$

The radial velocity $v_{r}$ is zero for radii smaller than $r_{0}$ and rises to an almost constant value on a small radial distance $\frac{\partial v_{r}}{\partial r} \sim v_{r} \delta\left(r-r_{0}\right)$. The equation of continuity becomes

$$
\frac{d}{d t} \ln n=-v_{r} \delta\left(r-r_{0}\right)
$$

where $\frac{d}{d t}=\frac{\partial}{\partial t}+v_{r} \frac{\partial}{\partial r}$. The solution is

$$
\ln n[r(t)]=\ln n\left[r\left(t_{0}\right)\right]-\int_{t_{0}}^{t} d t^{\prime} v_{r} \delta\left[r(t)-r_{0}\right]
$$

where the integral along the trajectory is the inverse of the Lagrangian derivation $d / d t$. The expression shows that the density is simply carried along the streamer, with a discontinuity in the small region at the foot of the streamer. This leads to a depression of density if the neighbor regions are not providing aflux of particles. We will show that there is an instability consisting of strong modulation of the density on the poloidal circle $r_{0}$ which can lead to accumulation of density in periodic poloidal positions. Although this instability and the formation of the streamer are independent, they can act concurrently, avoiding suppression of the streamer or its breaking up with further propagation as blob. This is the reason for which we examine now the poloidal modulation of the density when a conjunctural factor (the streamer) imposes a flow along $\theta$.

Assuming incompressibility $\boldsymbol{\nabla} \cdot \mathbf{v}=0$ in the small region around the streamer's foot we have $\delta v_{\theta} / \delta l_{\theta}=\delta v_{r} / \delta l_{r}$ with comparable variation scales $\delta l_{\theta} \sim \delta l_{r}$. The two velocities, $v_{\theta}$ and $v \equiv v_{r}$ are therefore related $v_{\theta} \sim v$. Accordingly, the acceleration of the streamer velocity $v$ along the radius induces an accelaration of the flow $v_{\theta}$ on the poloidal circle.

$$
\frac{\partial v}{\partial t} \sim \frac{\partial v_{\theta}}{\partial t}
$$


which simply means that the time variation of the velocity in the streamer is also the time variation of the poloidal dynamics.

From now on our considerations will be refering to the poloidal direction only, more precisely to a narrow region around the circle of radius $r_{0}$ where the hot streamer is born. As said before the acceleration on the radial direction of the flow in the hot streamer induces an acceleration of the aflux of particles, along the poloidal circle, $\partial v_{\theta} / \partial t$, toward the foot of the streamer. In this region the dynamics along the hot streamer, essentially radial, is coupled to the dynamics along the poloidal circle. If the main characteristic of the hot streamer is the contrast of temperature relative to the environment in which it advances, the dynamics along the poloidal region regards essentially the density. This is because the temperature on the circle is constant while the particles must flow toward the streamer 's foot to sustain the streamer flux and this generates density perturbation. We introduce the density $\rho$ on the circle and we have that in the region of perturbation induced by the streamer, $\rho=\rho_{0}$. The problem becomes one-dimensional, along the circle $r_{0}$, with the coordinate $y \equiv r_{0} \theta$ and we take the origin of $y$ at the axis of the streamer, i.e. $y=0$ at $\theta_{0}$. For uniformity, we change the notation, $v_{y} \equiv v_{\theta}$.

A new manifestation of the baroclinic effect takes place along the poloidal direction. We first remind the case of the streamer, where the baroclinic force arises from the contrast of temperature between the hot streamer and the environment, in combination with the gradient of the background density. Now the baroclinic effect arises from the perturbation of density along the poloidal direction, in combination with the gradient of the equilibrium pressure, $\boldsymbol{\nabla} p_{0}$, in which the gradient of the equilibrium temperature $T_{0}(r)$ is active. Assume that due to the flux of particles towards the foot of the streamer $y=0$ there is a perturbation in the form of a lump of density at this point,. We have, focusing our considerations on the left side of the hot streamer, $\partial \rho / \partial y>0$,

$$
\frac{\partial \boldsymbol{\omega}}{\partial t} \sim \frac{1}{\rho^{2}} \nabla \rho \times \nabla p_{0}=\frac{1}{\rho^{2}}\left(\frac{\partial \rho}{\partial y}\right)\left|\frac{d p_{0}}{d r}\right| \widehat{\mathbf{e}}_{z}
$$

The effect of this baroclinic force is to enhance the flow along $y$ toward $y=0$. Taking a distance on $y$ of perturbation of $\rho(y)$ of the scale as the radius of the rolls of convection, $R$, we have an estimation of the effect on velocity coming from the left to $y=0$, the axis of the streamer. There is acceleration

$$
\frac{\partial v_{y}}{\partial t} \sim R \frac{\partial \omega}{\partial t}=R\left[\frac{1}{\rho^{2}} \frac{\partial \rho}{\partial y}\left|\frac{\partial p_{0}}{\partial r}\right|\right]=R \frac{1}{\rho} \frac{\partial \rho}{\partial y}\left|\frac{\partial\left(T_{0} / m_{i}\right)}{\partial r}\right|
$$

and the balance of forces along $y$ is

$$
\rho\left(\frac{\partial v_{y}}{\partial t}+v_{y} \frac{\partial v_{y}}{\partial y}\right)=\rho\left(R \frac{1}{\rho} \frac{\partial \rho}{\partial y}\left|\frac{\partial\left(T_{0} / m_{i}\right)}{\partial r}\right|\right)
$$


We set

$$
\sigma \equiv R\left|\frac{\partial\left(T_{0} / m_{i}\right)}{\partial r}\right|=R\left|\frac{\partial v_{t h, i}^{2}}{\partial r}\right|
$$

with dimensions of squared velocity.

We will express the term in the right hand side as a derivation to $y$, such as to exhibit the structure of the force resulting from the gradient of a scalar pressure, which we will have to identify. This means to find a function $F(y)$ such as

$$
\rho\left(\sigma \frac{1}{\rho} \frac{\partial \rho}{\partial y}\right) \equiv-\frac{\partial}{\partial y} F(\rho)=-\frac{d F(\rho)}{d \rho} \frac{\partial \rho}{\partial y}
$$

We obtain

$$
\begin{aligned}
\frac{d F(\rho)}{d \rho} & =-\sigma \\
F(\rho) & =-\sigma \rho
\end{aligned}
$$

and this allows us to characterize the term arising from the baroclinic effect as a force produced by the $y$-gradient of a pressure

$$
\rho\left(\sigma \frac{1}{\rho^{2}} \frac{\partial \rho}{\partial y}\right) \equiv-\frac{\partial}{\partial y} \bar{p}
$$

where the equivalent pressure, as a scalar function whose gradient is the force, is identified as

$$
\bar{p}=-\sigma \rho
$$

and is negative. The equations of motion for an element of plasma situated on the poloidal circle at radius $r_{0}$ consists of the deformation displacements in the poloidal direction due to the force generated from the gradient of the scalar pressure

$$
\frac{\partial v_{y}}{\partial t}+v_{y} \frac{\partial v_{y}}{\partial y}=-\frac{1}{\rho} \frac{\partial}{\partial y} \bar{p}
$$

and the continuity equation

$$
\frac{\partial \rho}{\partial t}+\frac{\partial}{\partial y}\left(\rho v_{y}\right)=0
$$

The same problem has been treated by Ott [38] where the temperature is negative. The pressure in the present case is negative with linear dependence on the perturbation of the density. This is the typical $m=1$ case of the general treatment of Trubnikov and Zhdanov [39].

In the Appendix A we show, following Trubnikov and Zhdanov [39], how this system is solved. The solution has the following characteristic: the 
density on the poloidal circle has regions of broad, quasi-uniform magnitude separated by very high (spiky) accumulations. In the actual physical process the strong accumulations coincide with the initialization of the hot streamers and effectively support the flow of density they represent.

\subsection{Comment on the theory of the anomalous poly- tropic gas}

We comment on the applicability of the results obtained from the model of anomalous polytropic gas to the physical problem of breaking of the azimuthal symmetry of the density and flow in tokamak with generation of radial streamers and further of rolls of convection.

We have two distinct processes: (1) a hot stream of plasma leaving the region at radius $r_{0}$ and advancing in the colder environment at higher $r$, sustained by a baroclinic force. The baroclinic force is generated by the combined effect of the contrast of temperature between the streamer and the environment and the gradient of equilibrium density profile. It is a fast process and a density depletion around $r_{0}$ can appear. (2) an instability of the density profile along the poloidal direction at $r_{0}$, again sustained by a baroclinic effect. This is determined by the combination between the gradient of the perturbed density along $\theta$ and the gradient of the equilibrium pressure. The effect is generation of high accumulation of density at periodic positions along the poloidal direction.

These two processes are interacting: the tendency of accumulation of density at certain location on the poloidal circle supports the flow in the streamers. The decrease of the density at $r_{0}$ by the streamer flow is compensated by the aflux of density associated to the (Chaplygin gas - type) instability of the density on $\theta$. Observations and numerical simulations suggest that the streamers ("plumes" or "thermals") of hot fluid inhibit the instability on the transversal direction by the high rate of radial flow which sometimes lead to breaking-up of streamer from its original region and separation as a blob.

The system of equations describing the instability in the early phase can be reduced to an $1 D$ model of a gas with anomalous polytropic (the pressure is negative and $\bar{p} \sim-\sigma \rho)$. The solution consists of regions of broad and smooth variation of the variables $(\rho, v)$ and very localised, spiky regions of high concentration of density.

The instability of Chaplygin gas is fast: the streamers (which appear in the model as concentrations of density) are formed on a time scale given by 
the equation

$$
\frac{\Lambda}{\tau} \sim \sigma^{1 / 2}
$$

which results from the eigenmodes determined in the linearized system (the dispersion relation already shows this characteristic scales). Here $\Lambda$ and $\tau$ are typical wavelength and time scales of the Chaplygin instability on $y$. Let us take $\rho_{0}=m_{i} n \sim 10^{-8} \mathrm{~kg} / \mathrm{m}^{3}, R \sim a / 10 \sim 0.1(\mathrm{~m})$,

$$
\frac{d p_{0}}{d r} \sim \frac{1}{(a / 10)} n T=3 \times 10^{4}\left(\frac{J}{m^{4}}\right)
$$

for $n=10^{19}\left(\mathrm{part} / \mathrm{m}^{3}\right)$ and $T=2000(\mathrm{eV})$. Then

$$
\sigma^{1 / 2} \approx 5 \times 10^{5}\left(\frac{m}{s}\right)
$$

Now we estimate the distance between the centres of two neighbor rolls, taking the pattern consisting of $m=5$ convective rolls with their centres at $r_{0} \sim a / 3$, for a minor radius $a=1(m), \Lambda=2 \pi r_{0} / m=0.4(m)$. Then the time scale is

$$
\tau=\frac{\Lambda}{\sigma^{1 / 2}} \sim 10^{-6}(s)
$$

which is very fast. Moreover, this time scale is compatible with the time scale determined independently from the flow of the hot streamer, since from Eq.(10) we also obtain $\tau \sim 10^{-6} \ldots 10^{-5}(s)$.

\section{The poloidal velocity as the envelope of the rotations in the cells of convection (rolls)}

The peripheric velocity of the convection rolls is transferred to plasma at larger $r$ both by Reynolds stress and by collisional drag. On the other hand even the geometry of the flow around closed rolls leads to a continuous (but wavy) streamlines which can represent a continuous poloidal flow. We want to show how to obtain the envelope representing the connected poloidal flow arising from separated rolls having rotations in the same direction. Therefore we will restrict the treatment to the pure geometric properties of the ideal flow. The Reynolds stress and collisions will enhance the efficiency of producing a directed poloidal flow.

The treatment is based on complex formalism [40] for the plane flow. We consider the fluid in the complex $z$ plane with a set of vortices placed at 
different positions. We need the complex potential of the flow in the $z$ plane, $w(z)$. The problem is not solved directly in the physical plane of the flow but in a plane $\zeta$ connected with $z$ by a conformal transformation. The idea is to take in the $\zeta$-plane a structure which is standard (canonical). It consists of (1) the circle with the center in $\zeta=0$ and unit radius $|\zeta|=1$ and (2) a set of $M$ circles with centres $\delta_{j}$ and radii $q_{j}$ placed inside the circle $|\zeta|=1$.

By the conformal map $\zeta \rightarrow z(\zeta)$ this corresponds to $M+1$ fixed circular obstacles in the $z$ plane of the physical flow. Our rolls of convection are patches of rotating fluid for which it is only specified the circulation of the fluid around (i.e. the integral of the tangential velocity on the closed contour) $\gamma_{j}, j=1, M$. The fluid moves in the $z$ plane with the condition of keeping the given value $\gamma_{j}$ of the circulation at every circle $j$. This does not uniquely specify the flow. One needs the condition at infinity, far from the regions where the circular vortices are given.

After defining the geometry it is possible to construct the complex potential in the $\zeta$ plane. It is easily shown that it is

$$
w(\zeta)=-\frac{i}{2 \pi} \ln \left[\frac{\zeta-\alpha}{\left|\alpha^{*}\right|\left(\zeta-\frac{1}{\alpha^{*}}\right)}\right]-\frac{i}{2 \pi} \ln \zeta+\text { const }
$$

This function represents the flow around a unit vortex located in $\alpha \in \zeta$-plane and is constant on the circle bounding the large domain $|\zeta|=1$. The complex potential gives a circulation of -1 around the circle $|\zeta|=1, \oint_{|\zeta|=1} \mathbf{v} \cdot \mathbf{d} \mathbf{l}=-1$ where $\mathbf{v}$ is calculated from $w$ according to the formulas of the Appendix B.

To simplify, we take the geometry in the $z$-plane of the physical flow as consisting of three convection rolls represented by three disks with centers at $z_{-1}=-d, z_{0}=0, z_{+1}=+d$, with equal radii, $s$. The circulation around each vortex roll is $\gamma$.

In the plane $\zeta$ one has a circle of radius $|\zeta|=1$. This will be transformed by the conformal map $\zeta \rightarrow z$ in the circle with center at $z_{0}=0$, which is one of the fixed physical vortices (or rolls of convection), the central one. Two other circles are inside this $|\zeta|=1$ domain, located at $\delta_{-1}=-\delta, \delta_{+1}=+\delta$ and having equal radii $=q$. Now the geometry of the $\zeta$ plane is defined. One has to write the complex potential in the $\zeta$ plane.

To impose a particular circulation on the contour of the $j^{\text {th }}$ circular patch of vorticity (or obstacle) from the set of $M$ in the $z$-plane, one defines in the 
$\zeta$-plane the potential

$$
G_{j}(\zeta, \alpha)=-\frac{i}{2 \pi} \ln \left(\frac{\omega(\zeta, \alpha)}{|\alpha| \omega\left(\zeta, \theta_{j}\left(\frac{1}{\alpha^{*}}\right)\right)}\right) \text { for } j=1, \ldots, M
$$

The function that has been introduced is

$$
\theta_{j}(\zeta)=\delta_{j}+\frac{q_{j}^{2} \zeta}{1-\delta_{j}^{*} \zeta}
$$

is a Mobius map. Then $G_{j}(\zeta, \alpha)$ is the complex potential of a flow in the plane $\zeta$ generated by a vortex of unit strength +1 in the point $\zeta=\alpha$ and making a circulation -1 around the circle $\delta_{j}$ of radius $q$. This potential has zero circulation around any of the other $M-1$ circles in the $\zeta$ plane and

around the circle $|\zeta|=1$. By superposition $-\sum_{j=0, j=1, \ldots, M} \gamma_{j} G_{j}(\zeta, \beta)$, one obtains in the plane $\zeta$ the complex potential for the canonical geometry: $|\zeta|=$ $1, \delta_{-1}, \delta_{+1}$. Since we assume that the convection rolls have equal circulations $\gamma_{j}=\gamma$ for $j=1,2,3$, we obtain $w(\zeta)=-\gamma_{-} G_{-}(\zeta, \alpha)-\gamma_{0} G_{0}(\zeta, \alpha)-$ $\gamma_{+} G_{+}(\zeta, \alpha)$. This complex potential is in $\zeta$ plane and from here it must be carried back onto the physical $(z)$ plane, by a conformal transformation which requires numerical calculation. The resulting flow have streamlines that show formation of poloidal rotation as we depart progressively from the line where the centers of the vortices are located [40. Since this is a gemetrical property it means that the poloidal flow occurs simultaneously with the onset of convection rolls, i.e. on a very fast time scale.

\section{The effect of the fast increase of the ra- dial electric field and the acceleration of the toroidal flow of bananas}

The high density and temperature gradients (or both), supported by external sources, lead to formation of large scale cells of convection. Due to the background neoclassical rotation the rolls have all the same direction of rotation. The baroclinic term induces high peripheric velocities in the rolls and the envelope of these peripheric flows is a poloidal rotation. The rise of the poloidal velocity takes place on the same time scale as the onset of the convection rolls and this is governed by the baroclinic term acting on the initial hot stream. It is a fast process, similar to the onset of convection in the classical Rayleigh-Benard system. One important aspect should be 
underlined: the flow in the convection rolls (hence the poloidal rotation) is sustained by the gradients of the equilibrium temperature and/or the equilibrium density, which in turn are sustained by external sources. Therefore the flow in the rolls of convection (implicitely the induced poloidal velocity) is sustained by external sources. This completely changes the usual picture on the poloidal rotation, the damping due to the magnetic pumping is now balanced and overcommed by the external sources. This also suggests the existence of a threshold, which will be examined in a separate work.

The dynamics in the radial direction consists of charge separation and has weak effect on the force balance along this direction. We consider the approximate radial equilibrium of forces

$$
E_{r} \approx \frac{1}{|e| n} \frac{d p}{d r}+v_{T} B_{\theta}-v_{\theta} B_{T}
$$

as valid over the interval of time increase of $v_{\theta}$. In Appendix $\mathrm{C}$ we argue that the fast change of $v_{\theta}$ is mainly reflected in a change of the radial electric field $E_{r}$, on the same time scale, leading to the approximative balance

$$
\frac{\partial E_{r}}{\partial t} \approx-B_{T} \frac{\partial v_{\theta}}{\partial t}
$$

Since we have

$$
\frac{v_{t h, i}}{R q} \lesssim \frac{\partial}{\partial t} \ll \Omega_{c i}
$$

it results that the time change of the radial electric field will modify the motion of the bananas.

We are in the regime described by Hinton and Robertson [37] where during the motion of the particle on the banana orbit there is effective change of the radial electric field which acts on the particle. In the Ref. 37] the effect is found to be manifested in two ways. First, there is a steady radial motion of the bananas. Second, in each bounce period, there is an acceleration of the motion in the toroidal direction. The effects are due to the fact that (schematically) the particle feels a certain radial electric field on half of its orbit and a different radial electric field when it returns along the second half of the orbit.

The main assumption that has led us to this estimation is that

$$
\frac{\partial \omega}{\partial t}=\frac{v}{R} \frac{1}{\delta t}
$$

i.e. the baroclinic term acts all around the circular path of the rolled up flow in the cell and accelerates the rotation. The time scale is given by the time 
of rotation in the cell.

$$
\delta t \sim \frac{R}{v} \approx \frac{\left(R\left|L_{n}\right|\right)^{1 / 2}}{(\eta \lambda)^{1 / 2} v_{i, t h}}
$$

The time variation of the poloidal velocity is the time of acceleration

$$
\frac{v}{\delta t} \sim \eta \lambda \frac{v_{i, t h}^{2}}{\left|L_{n}\right|}
$$

and the time derivative of the radial electric field is

$$
\frac{\partial E_{r}}{\partial t} \approx \frac{\partial}{\partial t}\left(-v_{\theta} B_{T}\right) \sim-\eta \lambda \frac{v_{i, t h}^{2}}{\left|L_{n}\right|} B_{T}
$$

At every bounce the banana gets a toroidal acceleration [37]

$$
\delta u=\left(\frac{\partial E_{r}}{\partial t}\right) \frac{1}{B_{\theta}} \delta t_{\text {bounce }}
$$

where

$$
\begin{gathered}
\delta t_{\text {bounce }}=\frac{q R_{0}}{v_{i, t h}} \\
\delta u \sim-\eta \lambda \frac{v_{i, t h}^{2}}{\left|L_{n}\right|} B_{T} \frac{1}{B_{\theta}} \frac{q R_{0}}{v_{i, t h}}=-v_{i, t h} \eta \lambda \frac{B_{T}}{B_{\theta}} \frac{q R_{0}}{\left|L_{n}\right|}
\end{gathered}
$$

This kick of acceleration, leading to the change of the toroidal velocity of the trapped particle $\delta u$ is due to the variation of the radial electric field during the time interval the trapped ion makes a complete bounce. The number of bounces that a trapped particle makes in an interval of time $\Delta t$ is

$$
n=\frac{\Delta t}{\delta t_{\text {bounce }}}=\frac{\Delta t}{q R_{0} / v_{i, t h}}
$$

During the time interval $\Delta t$ the total change of the toroidal velocity of a trapped particle is

$$
\Delta u=n \delta u=-\Delta t v_{i, t h}^{2} \frac{1}{\left|L_{n}\right|} \eta \lambda \frac{B_{T}}{B_{\theta}}
$$

This change is substantial. On a time interval of one millisecond $\Delta t=10^{-3} \mathrm{~s}$, with $L_{n} \approx 5 \mathrm{~m}, B_{T} / B_{\theta} \sim 5, v_{i, t h}=10^{5} \mathrm{~m} / \mathrm{s}$ and taking as before $\eta=0.1$, $\lambda=0.1$ the change of the toroidal velocity of a single trapped particle is of the order of the thermal velocity. When the initial toroidal flow is opposite to this acceleration there is reversal of rotation. The energy requested for this change is provided, in our model, by the equilibrium gradients of temperature and density. 


\section{Discussion and summary}

There are other mechanisms that can sustain or modify the spontaneous toroidal rotation. The drift wave turbulence creates a statistical ensemble of fluctuating velocities and the coupling of the radial and poloidal components can have a non-zero correlation (Reynolds stress) with spatial dependence. The divergence of this correlation is a driving force for the rotation. Due to the long correlation length in the toroidal direction, the Reynolds stress is efficient in the poloidal plane and in particular is able to induce zonal flows. If it overcomes the magnetic damping it can induce poloidal rotation and so generate a non-vanishing $\partial E_{r} / \partial t$ and the subsequent neoclassical polarization. The latter modifies the toroidal precession, i.e. the plasma toroidal rotation. A detailed analysis of this connection should be made, with focus on transitory processes, as observed in Alcator C-Mode [1]. However few aspects can be discussed qualitatively. Since it is based on a statistical ensemble the Reynolds stress needs a strong poloidal-symmetry breaking mechanism otherwise the average is zero. Indeed the symmetry breaking can exist, analogous to the flows arising in the Rayleigh-Benard experiments after higher order bifurcations (flows are called "winds"). However their manifestation is slower that all the other time scales: conduction, convection. It is difficult to see how the onset of a poloidal flow via only Reynolds stress can take place on time scales less than milliseconds, if the background consists of random drift waves. On the other hand it is well established that large scale convective flows have much higher Reynolds stress than the $2 D$ quasiisotropic turbulence. This is partly the reason for the present investigation, where we precede any effect of change of the rotation by the onset of a chain of convection cells. We further note one aspect that results from the combined effect of convective cells and the Stringer drive and compare it with the Reynolds stress. A chain of convective cells (with either unidirectional or alternating vorticity direction) induces a periodic modulation of the local rate of transport. The neoclassical Stringer effect converts this modulation into an effective poloidal torque, possibly higher than the damping due to magnetic pumping. In a similar case but in the absence of convection cells [41], the ratio of the magnitude of the Reynolds stress over the Stringer drive was found small. The presence of convective cells enhances both the Reynolds stress and the Stringer drive. Further study is necessary for a correct comparison of magnitudes.

We have proposed a phenomenological model for the reversal of the toroidal rotation in tokamak. The model assembles effects that separately are known to appear in tokamak plasma and have been studied both exper- 
imentally and theoretically. The generation of convection structures with flows that are quasi-coherent on large scales in the meridional section is in general admitted as a particularly effective way of transport of angular momentum and of energy across the magnetic field. We consider the situation of cells of convection with the same sense of rotation flow, disposed around the magnetic axis in the region extending from the core plasma to the confining zone, a structure that is compatible with the basic neoclassical flow. It is also inspired by atmospheric and protoplanetary flows where such distribution occurs in hydrodynamic regimes very similar to the one in tokamak. The convection is sustained by equilibrium gradients of density and temperature (which in turn are fed by external sources) and this drives the poloidal velocity which is generated by the combined, unidirectional, peripheric flows of the cells. Then the decay due to parallel viscosity (magnetic pumping) can be overcomed. The time scales are short due to the baroclinic term which accounts for the generation of vorticity. The fast increase of the poloidal velocity induces a fast variation of the radial electric field, on a time scale that is comparable with the bounce time. On part of the banana trajectory the ions see a magnitude of the radial electric field and on rest of the trajectory they see a different magnitude. This produces both radial motion and the change of velocity in toroidal direction. The collisions will spread this drive to the rest of the ion population.

The principal merits of this theoretical explanation is that it provides a connection between poloidal and toroidal rotation. Usually the direction of influence is reversed and based of instabilities: the sheared parallel flow induces instabilities whose turbulent Reynolds stress acts to generate and sustain the poloidal rotation against the magnetic pumping decay [42], [43]. This mechanism is active in situation where the turbulence is known to induce poloidal flows acting as internal transport barriers, while the basic flow is observed to be toroidal. It can interfere with the generation of convective structures, for example in formation of Goertler vortices [44], etc. In the present case the poloidal flow is a geometric consequence of the flow in the convection structures. The driving effect of the poloidal flow on the toroidal flow has a fast transient phase which is decissive since it involves neoclassical polarization on the time scale of the bounce on bananas.

The different components of this phenomenology appear to be compatible and to combine in a structure which is logically coherent. Future work is needed for a more detailed examination of this theory.

Acknowledgement 1 Work supported partially by the Contracts BS-2 and $B S-14$ of the Association EURATOM - MEdC Romania. The views presented here do not necessarly represent those of the European Commission. 


\section{A Appendix. The Chaplygin gas instability}

\section{A.1 The formalism for the Chaplygin gas instability}

We will linearize the equations (27) and (28), i.e.

$$
\frac{\partial \rho}{\partial t}+\frac{\partial}{\partial y}\left(\rho v_{y}\right)=0
$$

and

$$
\frac{\partial v_{y}}{\partial t}+v_{y} \frac{\partial v_{y}}{\partial y}=-\frac{1}{\rho} \frac{\partial}{\partial y} \bar{p}
$$

around the state $\rho=\rho_{0}, v_{y}=0$ and obtain

$$
\begin{aligned}
\frac{\partial}{\partial t}\left(\rho_{0}+\widetilde{\rho}\right)+\frac{\partial}{\partial y}\left[\left(\rho_{0}+\widetilde{\rho}\right) \widetilde{v}_{y}\right] & =0 \\
\frac{\partial \widetilde{v}_{y}}{\partial t}+\widetilde{v}_{y} \frac{\partial \widetilde{v}_{y}}{\partial y} & =-\frac{1}{\rho_{0}+\widetilde{\rho}} \frac{\partial}{\partial y}[-\sigma \widetilde{\rho}]
\end{aligned}
$$

We have

$$
\begin{aligned}
\frac{\partial \widetilde{\rho}}{\partial t}+\rho_{0} \frac{\partial \widetilde{v}_{y}}{\partial y} & =0 \\
\frac{\partial \widetilde{v}_{y}}{\partial t} & =-\frac{1}{\rho_{0}}\left(1-\frac{\widetilde{\rho}}{\rho_{0}}\right) \frac{\partial}{\partial y}(-\sigma \widetilde{\rho}) \approx \frac{1}{\rho_{0}} \sigma \frac{\partial \widetilde{\rho}}{\partial y}
\end{aligned}
$$

Now we take $\widetilde{\rho} \sim \exp [i(k y-\omega t)]$ and $\widetilde{v}_{y} \sim \exp [i(k y-\omega t)]$ and inserting in the linearized equations we get

$$
-i \omega \widetilde{\rho}+\rho_{0} i k\left[-\frac{1}{i \omega} \frac{\sigma}{\rho_{0}} i k \widetilde{\rho}\right]=0
$$

or

$$
\omega= \pm i k(\sigma)^{1 / 2}
$$

The positive imaginary part indicates instability, similar to the RayleighTaylor case.

The numerical solution of the system of equations [38] shows that there are broad regions of weak variation and very peaked accumulations, which evolve to singular spatial spikes.

The equations in Ref.[39] page 143 are relevant to the present case, for our negative pressure

$$
\bar{p}=-\sigma \rho
$$


If we introduce this expression in the equation of motion we obtain

$$
\frac{\partial v_{y}}{\partial t}+v_{y} \frac{\partial v_{y}}{\partial x}=-\frac{1}{\rho} \frac{\partial}{\partial y} \bar{p}=\frac{1}{\rho_{0}+\widetilde{\rho}} \frac{\partial}{\partial y}(\sigma \widetilde{\rho})
$$

and the system can be rewritten in terms of two variables $\widetilde{\rho}$ and $v_{y}$.

$$
\begin{aligned}
\frac{\partial \widetilde{\rho}}{\partial t}+\rho_{0} \frac{\partial v_{y}}{\partial y}+\frac{\partial}{\partial y}\left(\widetilde{\rho} v_{y}\right) & =0 \\
\frac{\partial v_{y}}{\partial t}+v_{y} \frac{\partial v_{y}}{\partial y} & =\frac{\sigma}{\rho_{0}} \frac{\partial}{\partial y} \widetilde{\rho}
\end{aligned}
$$

The equations belong to the elliptic type and therefore they do not have running waves. These equations correspond to the case $m=1$ in the general treatment made by Trubnikov and Zhdanov and it is shown that they lead to the Laplace equation.

We now try to apply the hodograph transformation to the equations of our case. Instead of the variables $\widetilde{\rho}(y, t)$ and $v_{y}(y, t)$ we will use the inverted variables $y\left(\widetilde{\rho}, v_{y}\right)$ and $t\left(\widetilde{\rho}, v_{y}\right)$ with the relationships between the derivatives

$$
\begin{aligned}
& \frac{\partial v_{y}}{\partial t}=\frac{1}{w} \frac{\partial y}{\partial \widetilde{\rho}}, \quad \frac{\partial v_{y}}{\partial y}=-\frac{1}{w} \frac{\partial t}{\partial \widetilde{\rho}} \\
& \frac{\partial \widetilde{\rho}}{\partial t}=-\frac{1}{w} \frac{\partial y}{\partial v_{y}} \quad, \quad \frac{\partial \widetilde{\rho}}{\partial y}=\frac{1}{w} \frac{\partial t}{\partial v_{y}}
\end{aligned}
$$

The Jacobian of the transformation is

$$
w=\frac{\partial y}{\partial \widetilde{\rho}} \frac{\partial t}{\partial v_{y}}-\frac{\partial y}{\partial v_{y}} \frac{\partial t}{\partial \widetilde{\rho}}
$$

Now we substitute these equations in the system, to make the transformation to the inverted variables $(y, t)$ instead of $\left(\widetilde{\rho}, v_{y}\right)$.

$$
\begin{aligned}
& \frac{\partial \widetilde{\rho}}{\partial t}+\rho_{0} \frac{\partial v_{y}}{\partial y}+\frac{\partial}{\partial y}\left(\widetilde{\rho} v_{y}\right) \\
= & -\frac{1}{w} \frac{\partial y}{\partial v_{y}}+\rho_{0}\left(-\frac{1}{w} \frac{\partial t}{\partial \widetilde{\rho}}\right)+\frac{1}{w} \frac{\partial t}{\partial v_{y}} v_{y}+\widetilde{\rho}\left(-\frac{1}{w} \frac{\partial t}{\partial \widetilde{\rho}}\right)=0
\end{aligned}
$$

We separate the derivative $\partial y / \partial v_{y}$

$$
\frac{\partial y}{\partial v_{y}}=-\left(\rho_{0}+\widetilde{\rho}\right) \frac{\partial t}{\partial \widetilde{\rho}}+v_{y} \frac{\partial t}{\partial v_{y}}
$$


Making the same substitution in the second equation we have

$$
\begin{aligned}
\frac{\partial v_{y}}{\partial t}+v_{y} \frac{\partial v_{y}}{\partial y} & =\frac{\sigma}{\rho_{0}} \frac{\partial}{\partial y} \widetilde{\rho} \\
\frac{1}{w} \frac{\partial y}{\partial \widetilde{\rho}}+v_{y}\left(-\frac{1}{w} \frac{\partial t}{\partial \widetilde{\rho}}\right) & =\frac{\sigma}{\rho_{0}} \frac{1}{w} \frac{\partial t}{\partial v_{y}}
\end{aligned}
$$

and we separate the derivative $\partial y / \partial \widetilde{\rho}$ from this equation

$$
\frac{\partial y}{\partial \widetilde{\rho}}=v_{y} \frac{\partial t}{\partial \widetilde{\rho}}+\frac{\sigma}{\rho_{0}} \frac{\partial t}{\partial v_{y}}
$$

Now we have two partial derivatives of $y$ to the two new variables $\left(\widetilde{\rho}, v_{y}\right)$. We impose the condition of compatibility of the mixed second order derivatives

$$
\frac{\partial}{\partial \widetilde{\rho}} \frac{\partial y}{\partial v_{y}}=\frac{\partial}{\partial v_{y}} \frac{\partial y}{\partial \widetilde{\rho}}
$$

which leads to

$$
\frac{1}{\rho_{0}+\widetilde{\rho}} \frac{\partial}{\partial \widetilde{\rho}}\left[\left(\rho_{0}+\widetilde{\rho}\right)^{2} \frac{\partial t}{\partial \widetilde{\rho}}\right]+\frac{\sigma}{\rho_{0}} \frac{\partial^{2} t}{\partial v_{y}^{2}}=0
$$

To go further (and show that this equation leads to the Laplace equation) we make another substitution of variables

$$
r \equiv\left(\rho_{0}+\widetilde{\rho}\right)^{1 / 2} \quad, \quad z \equiv \frac{1}{2} \frac{v_{y}}{\sqrt{\sigma / \rho_{0}}}
$$

Then

$$
\rho_{0}+\widetilde{\rho}=r^{2} \quad, \quad v_{y}=\alpha z
$$

where $\alpha \equiv 2 \sqrt{\sigma / \rho_{0}}$ and the change of variables implies

$$
\begin{aligned}
\frac{\partial}{\partial r} & =\frac{\partial}{\partial \widetilde{\rho}} \frac{\partial \widetilde{\rho}}{\partial r}=2 r \frac{\partial}{\partial \widetilde{\rho}} \\
\frac{\partial}{\partial z} & =\frac{\partial}{\partial v_{y}} \frac{\partial v_{y}}{\partial z}=\alpha \frac{\partial}{\partial v}
\end{aligned}
$$

or

$$
\frac{\partial}{\partial \widetilde{\rho}}=\frac{1}{2 r} \frac{\partial}{\partial r} \quad, \quad \frac{\partial}{\partial v_{y}}=\frac{1}{\alpha} \frac{\partial}{\partial z}
$$

Inserting in the equation, we have

$$
\frac{1}{\rho_{0}+\widetilde{\rho}} \frac{\partial}{\partial \widetilde{\rho}}\left[\left(\rho_{0}+\widetilde{\rho}\right)^{2} \frac{\partial t}{\partial \widetilde{\rho}}\right]+\frac{\sigma}{\rho_{0}} \frac{\partial^{2} t}{\partial v_{y}^{2}}=0
$$


which can be rewritten

$$
\frac{\partial^{2} t}{\partial r^{2}}+\frac{3}{r} \frac{\partial t}{\partial r}+\frac{\partial^{2} t}{\partial z^{2}}=0
$$

Following the general procedure (of Trubnikov and Zhdanov) we make the substitution

$$
\psi \equiv r t
$$

and replace the variable $t$ by $\psi$

$$
\begin{aligned}
t & =\frac{\psi}{r} \\
\frac{\partial t}{\partial r} & =-\frac{1}{r^{2}} \psi+\frac{1}{r} \frac{\partial \psi}{\partial r} \\
\frac{\partial^{2} t}{\partial r^{2}} & =\frac{2}{r^{3}} \psi-\frac{1}{r^{2}} \frac{\partial \psi}{\partial r}-\frac{1}{r^{2}} \frac{\partial \psi}{\partial r}+\frac{1}{r} \frac{\partial^{2} \psi}{\partial r^{2}}
\end{aligned}
$$

and we obtain

$$
\frac{\partial^{2} \psi}{\partial r^{2}}+\frac{1}{r} \frac{\partial \psi}{\partial r}-\frac{1}{r^{2}} \psi+\frac{\partial^{2} \psi}{\partial z^{2}}=0
$$

This is the Laplace equation for a potential of the form

$$
\Psi(r, \varphi, z)=\psi(r, z) \cos \varphi
$$

\section{A.2 The solution of the Laplace equation}

In Ref.[39] it is explained the so-called "Evolutionary principle ", a restriction placed on the solutions of the Laplace equation: the physically useful solutions must satisfy the condition that, returning in time to the infinite past $t \rightarrow-\infty$ the function $\Psi$ or $(\rho, v)$ must take the values of the equilibrium profile. This means $\rho \rightarrow \rho_{0}$ and $v_{y} \rightarrow 0$, or, equivalently, $r=1$ and $z=0$. In accord with the last form of the Laplacean expressed in variables $(r, \varphi, z)$ this corresponds to a circle or radius 1 in the plane $z=0$. All the initial conditions at infinite past must be taken on a thin region around this circle. Since the Laplace equation also describes the electrostatic field, Trubnikov and Zhdanov exploit this analogy by formulating the problem as the solution of an electrostatic problem in space, generated by a certain distribution of charges on the circle $r=1$ and $z=0$. The solutions that are admissible must be singular on this circle. The distribution of charges is equivalently described in terms of multipoles. Trubnikov and Zhdanov show that only two multipoles are useful for applications: the Coulomb distribution and the dipole distribution of charges on the circle. The first provides 
periodic solutions in real (i.e. physical) space. The "2-multipole" (dipole) generates three types of solutions in the physical space: the hill, the well and the doublet consiting of well+hill.

The solution of the Laplace equation in the space $(r, \varphi, z)$ with boundary conditions given on the circle $(r=1, z=0)$ requires to assume the toroidal geometry and therefore to introduce the system of coordines

$$
r=\lambda \sinh \xi, \quad z=\lambda \sin \eta
$$

where $\lambda=(\cosh \xi+\cos \eta)^{-1}$ and the metric is

$$
d r^{2}+d z^{2}=(\lambda d \xi)^{2}+(\lambda d \eta)^{2}
$$

The Lamé coefficients are $h_{\xi}=\lambda, h_{\varphi}=r=\lambda \sinh \xi$ and $h_{\eta}=\lambda$. The Laplace equation is

$$
\frac{\partial}{\partial \xi}\left(\lambda \sinh \xi \frac{\partial \Psi}{\partial \xi}\right)+\frac{\partial}{\partial \eta}\left(\lambda \sinh \xi \frac{\partial \Psi}{\partial \eta}\right)+\frac{\lambda}{\sinh \xi} \frac{\partial^{2} \Psi}{\partial \varphi^{2}}=0
$$

In our particular case, which corresponds to $m=1$ of the general theory, we must seek solutions of the type $\Psi=\psi(r, z) \cos \varphi$ and after the substitution

$$
\psi(r, z)=r^{-1 / 2} f
$$

the amplitude is written as a series of terms with separated variables

$$
f=\sum_{n=0}^{\infty} f_{n}(\xi) \cos (n \eta)
$$

Now it is introduced a new variable instead of $\xi$

$$
\chi \equiv \operatorname{coth} \xi
$$

and this transforms the Laplace equation into the equation for Legendre polynomials

$$
\frac{d}{d \chi}\left[\left(1-\chi^{2}\right) \frac{d f_{n}}{d \chi}\right]+\left[\nu(\nu+1)-\frac{n^{2}}{1-\chi^{2}}\right] f_{n}=0
$$

for $\nu \equiv-3 / 2$. The solutions are, in general

$$
P_{\nu}^{n}(\xi) \text { and } Q_{\nu}^{n}(\xi)
$$

However only the solutions $Q_{\nu}^{n}(\xi)$ must be retained since they have the property to become singular on the circle $r=1$ at $t \rightarrow-\infty$.

The solution is characterised by large regions where the function is smooth with weak variation and local spikes which evolve in time to singularity. The distribution of density is then compatible with the independent creation of streams of plasma which require strong density flow. 


\section{B Appendix. The complex potential of flow of a system of circular vorticity patches}

The flow is described by a complex function named complex potential $w$. It is composed from:

1. the real part, $\varphi(x, y)$, is the potential of the flow, i.e. the function from which the components of the velocity are derived

$$
v_{x}=\frac{\partial \varphi}{\partial x} \quad, \quad v_{y}=\frac{\partial \varphi}{\partial y}
$$

This is the simple expression of the physical fact that the flow is potential, i.e. it is derived as the gradient of the scalar function $\mathbf{v}=\boldsymbol{\nabla} \varphi$. The flow is therefore irrotational, the vorticity is zero $\boldsymbol{\omega}=\boldsymbol{\nabla} \times(\boldsymbol{\nabla} \varphi) \equiv \mathbf{0}$.

2. the imaginary part, $\psi(x, y)$ which is the streamfunction and is related to the potential function $\varphi$ by the fact that it also gives the components of the velocity

$$
v_{x}=-\frac{\partial \psi}{\partial y}, v_{y}=\frac{\partial \psi}{\partial x}
$$

Then

$$
\frac{\partial \varphi}{\partial x}=-\frac{\partial \psi}{\partial y} \quad, \quad \frac{\partial \varphi}{\partial y}=\frac{\partial \psi}{\partial x}
$$

which are the Riemann-Cauchy conditions for holomorphy of the complex potential $w(x, y)$. The lines of streamfunction $\psi(x, y)$ are perpendicular on the lines of the potential $\varphi$. The velocity is tangent to the lines $\psi(x, y)=$ const.

Then the complex potential is

$$
\begin{aligned}
w(x, y) & =\varphi(x, y)+i \psi(x, y) \text { or } \\
w(z) & =\varphi(z)+i \psi(z)
\end{aligned}
$$

Since $\varphi$ and $\psi$ are the real and imaginary part of a holomorphic function (in a region of holomorphicity) both are harmonic functions

$$
\begin{aligned}
& \Delta \varphi=0 \\
& \Delta \psi=0 \text { in the regions outside vortices }
\end{aligned}
$$

and the flow has no vorticity with the exception of the closed regions where the circulations is defined, $\gamma_{j}$. 


\section{Appendix. The fast increase of the poloidal velocity}

The problem consists of calculation of the radial polarization current when the poloidal velocity is strongly forced. The process has two stages: the first is the onset of the poloidal rotation, as envelope of the peripheric flows of a chain of convection cells in the meridional plane; the second is the sustainement of the poloidal rotation against magnetic damping and viscosity. The first stage is very fast, as is suggested by the similarity with the Rayleigh-Benard bifurcation conduction/convection and as is confirmed by the evaluation of the drive of the streamers by the baroclinic term in the vorticity equation. In this stage the rise of the poloidal flow generates a time-varying radial polarization electric field which acts on trapped ions on the time scale of their bounce producing a toroidal acceleration. The work done by the radial electric field on trapped ions is supported by the energy coming from the flow in the convection cells and this one is in turn supported by gradients of equilibrium plasma parameters (ultimetely from external input).

In general the radial projection of the ion momentum connects terms that contain the poloidal velocity $v_{y}^{i}$, the toroidal velocity $v_{z}^{i}$, the radial electric field $E_{r}$ and the diamagnetic term $\sim d p_{i 0} / d r$. Since now the poloidal velocity is forced, it is important to see which of the other terms will get the maximum effect on the short time scale of the onset of the convection rolls. Based on reasonable assumptions we show that the drive of $\partial v_{y}^{i} / \partial t$ goes primarly to the radial electric field $\left(\partial E_{r} / \partial t\right.$, the polarization). This further justifies to look to its effect on trapped ions.

The estimations of the important effects will proceed along (and it is part of) the derivation of the initial value problem for the poloidal velocity, as shown in Ref. [45] The radial current results from the Ampere's law

$$
\nabla \times\left.\mathbf{B}\right|_{x}=0=\mu_{0} j_{x}+\varepsilon_{0} \mu_{0} \frac{\partial E_{x}}{\partial t}
$$

where

$$
j_{x}(t)=-e n v_{x}^{e}+e n v_{x}^{i}
$$

We use a slab geometry where the coordinates are: $x \equiv r$ with $\widehat{\mathbf{e}}_{x} \equiv$ $-\widehat{\mathbf{e}}_{r} \quad$ (toward the plasma interior), $y \equiv r \theta$ with $\widehat{\mathbf{e}}_{y} \equiv \widehat{\mathbf{e}}_{\theta}$ (poloidal), and $z \equiv R \varphi$ with $\widehat{\mathbf{e}}_{z} \equiv \widehat{\mathbf{e}}_{\varphi}$ (toroidal). The conservation of the ion momentum in the radial direction is

$$
n_{i} m_{i}\left(\frac{\partial v_{x}^{i}}{\partial t}+v_{y}^{i} \frac{\partial v_{x}^{i}}{\partial y}\right)=-\frac{\partial p_{i 0}}{\partial x}+e n_{i} E_{x}+e n_{i} v_{y}^{i} B_{z}-e n_{i} v_{z}^{i} B_{y}+R_{x}
$$


The lines of flow in the poloidal direction are wiggly due to the discrete chain of rolls but on the average the term with the poloidal $(y)$ derivative of the radial $v_{x}^{i}$ velocity vanishes. In the following we we neglect the resistive terms.

$$
v_{y}^{i}=\frac{m_{i}}{e B_{z}} \frac{\partial v_{x}^{i}}{\partial t}-\frac{E_{x}}{B_{z}}+\frac{B_{y}}{B_{z}} v_{z}^{i}+\frac{1}{n_{i} m_{i} \Omega_{i}} \frac{\partial p_{i 0}}{\partial x}
$$

the last term being the diamagnetic flow contribution. The equation for the poloidal velocity $v_{y}^{i}$ is indicative for the effect that can result from the fast time variation, when the poloidal flow is strongly forced by convection rolls. The time variation of $v_{y}^{i}$ can induce a time variation of the diamagnetic term, but this can be considered small since the change of the equilibrium profile of the density involves diffusive time scale. The change of the density due to the perturbation (i.e. the convection cells) appears here in second order and will be neglected.

Further, we have to bear in mind our main objective, which is to show the possibility of coupling of the poloidal and toroidal rotations via the neoclassical polarization, acting on trapped ions. For this reason we can postpone the inclusion of the direct (i.e. not mediated by neoclassical polarization) effect of $\partial v_{y}^{i} / \partial t$ upon the term $\frac{B_{y}}{B_{z}} v_{z}^{i}$. The relative importance of this term will be discussed at the end of this Appendix. Then we will use the approximations

$$
v_{y}^{i} \approx-\frac{E_{x}}{B_{z}}+\frac{m_{i}}{e B_{z}} \frac{\partial v_{x}^{i}}{\partial t}
$$

The conservation of the ion momentum in the poloidal direction is

$$
n_{i} m_{i}\left(\frac{\partial v_{y}^{i}}{\partial t}+v_{x}^{i} \frac{\partial v_{y}^{i}}{\partial x}\right)=-\frac{\partial p_{i 0}}{\partial y}+e n_{i} E_{y}-e n_{i} v_{x}^{i} B_{z}+R_{y}
$$

Since this calculation is made on the points along the poloidal circle where the convection rolls are closed the radial ion velocity $v_{x}^{i}$ on this circle and the nonlinear term $v_{x}^{i} \frac{\partial v_{y}^{i}}{\partial x}$ are small. Also the poloidal $(y)$ components of the electric field $E_{y}$ and of the gradient of the pressure are vanishingly small compared with the rest of the terms (actually they are neoclassical corrections related to trapping and toroidal viscosity, which are here comparatively small). It results

$$
v_{x}^{i}=-\frac{1}{\Omega_{i}}\left(\frac{\partial v_{y}^{i}}{\partial t}\right)\left[1+\frac{1}{\Omega_{i}}\left(\frac{\partial v_{y}^{i}}{\partial x}\right)\right]^{-1}
$$

Now we use the Ampere's law

$$
-e n_{e} v_{x}^{e}=-e n_{i} v_{x}^{i}-\varepsilon_{0} \frac{\partial E_{x}}{\partial t}
$$


In this expression we insert the radial electric field from Eq.(C.5) and the radial ion's velocity from Eq.(C.7). We obtain a single equation for the poloidal ion velocity

$$
\begin{aligned}
-e n v_{x}^{e}= & \frac{e n}{\Omega_{i}}\left(\frac{\partial v_{y}^{i}}{\partial t}\right) \frac{1}{U}+\varepsilon_{0} B_{z}\left(\frac{\partial v_{y}^{i}}{\partial t}\right) \\
& +\frac{\varepsilon_{0} m_{i}}{e \Omega_{i}}\left(\frac{\partial^{3} v_{y}^{i}}{\partial t^{3}}\right) \frac{1}{U}-\frac{2 \varepsilon_{0} m_{i}}{e \Omega_{i}^{2}}\left(\frac{\partial^{2} v_{y}^{i}}{\partial t^{2}}\right)\left(\frac{\partial^{2} v_{y}^{i}}{\partial x \partial t}\right) \frac{1}{U^{2}} \\
& -\frac{2 \varepsilon_{0} m_{i}}{e \Omega_{i}^{3}}\left(\frac{\partial v_{y}^{i}}{\partial t}\right)\left(\frac{\partial^{2} v_{y}^{i}}{\partial x \partial t}\right)^{2} \frac{1}{U^{3}}-\frac{\varepsilon_{0} m_{i}}{e \Omega_{i}^{2}}\left(\frac{\partial v_{y}^{i}}{\partial t}\right)\left(\frac{\partial^{3} v_{y}^{i}}{\partial x \partial t^{2}}\right) \frac{1}{U^{2}}
\end{aligned}
$$

with the notation $U \equiv 1+\frac{1}{\Omega_{i}}\left(\frac{\partial v_{y}^{i}}{\partial x}\right)$. For the phase of the acceleration the variation on $x$ (radial) is not relevant and a simplified form is obtained

$$
-e n_{e} v_{x}^{e}=\varepsilon_{0} B_{z}\left(1+\frac{c^{2}}{v_{A}^{2}}\right) \frac{\partial v_{y}^{i}}{\partial t}+\varepsilon_{0} \frac{m_{i}^{2}}{e^{2} B_{z}} \frac{\partial^{3} v_{y}^{i}}{\partial t^{3}}
$$

The calculations must be repeated for electrons, to derive the expression of $v_{x}^{e}$. The relevant terms have however factors of $\Omega_{e}^{-1}$ and since $\Omega_{e} \gg \Omega_{i}$ the electron current can be neglected

$$
\left(\varepsilon_{\perp} / \varepsilon_{0}\right) \frac{\partial v_{y}^{i}}{\partial t}+\frac{1}{\Omega_{i}^{2}} \frac{\partial^{3} v_{y}^{i}}{\partial t^{3}} \approx 0
$$

The quantity $\varepsilon_{\perp}=\varepsilon_{0}\left(1+c^{2} / v_{A}^{2}\right)$ is the effective plasma dielectric in the direction transversal to the strong magnetic field. The initial condition is the acceleration of the poloidal flow resulting from forcing at the onset of the convection, $K \equiv d V_{y} / d t$. As said before, this process (similar to RB bifurcation) is expected to be very fast but its details are still beyond analytical description.

$$
\left(\frac{1}{\Omega_{i}^{2}} \frac{\partial^{2}}{\partial t^{2}}+\frac{\varepsilon_{\perp}}{\varepsilon_{0}}\right) \frac{\partial v_{y}^{i}}{\partial t}+K \Theta(t)=0
$$

where $\Theta(t)$ is the Heaviside function. With the time scaled $t \rightarrow t \Omega_{c}$ the equation becomes

$$
\ddot{w}+b w=-\bar{K} \Theta(t)
$$

Here $w(t) \equiv \partial v_{y}^{i} / \partial t$ and the dot means derivative to the scaled time variable. The notations are $b=\varepsilon_{\perp} / \varepsilon_{0}$ and $\bar{K} \equiv \Omega_{c}^{-1} K$. The solution, obtained by Laplace transform [45] is

$w(t)=\frac{-\bar{K}}{\varepsilon_{\perp} / \varepsilon_{0}}+\left(w_{0}-\frac{-\bar{K}}{\varepsilon_{\perp} / \varepsilon_{0}}\right) \cos \left[\left(\varepsilon_{\perp} / \varepsilon_{0}\right)^{1 / 2} t\right]+w_{1}\left(\frac{1}{\varepsilon_{\perp} / \varepsilon_{0}}\right)^{1 / 2} \sin \left[\left(\varepsilon_{\perp} / \varepsilon_{0}\right)^{1 / 2} t\right]$ 
For simplicity we have not included the terms that induce the decay of the oscillations [45]. The initial conditions are given for the first and the second derivatives of the velocity, respectively $w_{0}=w(t=0)$, and $w_{1}=\dot{w}(t=0)$. From the definition of $K$ we have $w_{0}=w(t=0)=\Omega_{c}^{-1} K=\frac{1}{\Omega_{i}}\left(\frac{d V_{y}}{d t}\right)_{t=0}$.

The non-oscillatory part of acceleration $\bar{w}(t)=\frac{-\bar{K}}{\varepsilon_{\perp} / \varepsilon_{0}}$ results from estimations made in the Section 2. During the onset of convection cells the poloidal velocity reach values comparable to the ion thermal velocity $\sim 10^{5}(\mathrm{~m} / \mathrm{s})$ in the characteristic time of advancement of the streamer, $10^{-6}(\mathrm{~s})$. Then the initialization is $\left(d V_{y} / d t\right)_{t=0} \sim 10^{11}\left(\mathrm{~m} / \mathrm{s}^{2}\right)$ and the non-oscillatory part has the order of magnitude

$$
\left(\frac{d V_{y}}{d t}\right)_{t=0} \frac{1}{\varepsilon_{\perp} / \varepsilon_{0}} \sim 10^{8}\left(\mathrm{~m} / \mathrm{s}^{2}\right)
$$

Now we can return to Eq.(C.4) and use this estimation to compare the terms that are supposed to share the effect of the fast increase of the poloidal velocity. The only term that may preoccupate contains the toroidal velocity. Taking as reference the experimental observation [2] the toroidal velocity is of the order of $10^{4}(\mathrm{~m} / \mathrm{s})$ and any change takes place on a time scale of fractions of a second. This means that the observed toroidal acceleration is substantially slower than Eq.(C.12). Besides, this term is multiplied by the small ratio $B_{y} / B_{z} \ll 1$. We conclude that the fast time variation of the poloidal velocity can only be followed by the radial electric field, which justifies Eq.(37).

\section{References}

[1] Rice J.E. et al. 2011 Nucl. Fusion 51083005

[2] Rice J.E. et al. 2005 Nucl. Fusion 45251

[3] Rice J.E. et al. 2001 Nucl. Fusion 41277

[4] Rice J.E. et al. 2004 Nucl. Fusion 44379

[5] Rice J.E. et al. 2003 Nucl. Fusion 43781

[6] Bortolon A. et al. 2006 Phys. Rev. Letters 97235003

[7] Fiore C.L. et al. 2010 Nucl. Fusion 50064008

[8] Rice J.E. et al. 2004 Phys. Plasmas 112427 
[9] Chandraskhar S. 1981 Hydrodynamic and Hydromagnetic Stability Dover, New York, USA

[10] Lappa M. 2010 Thermal convection: patterns, evolution and stability Wiley, Chichester, UK

[11] Kraichnan R.H. and Montgomery D. 1980 Rep. Prog. Phys. 4335

[12] Corcos G.M. and Sherman S.F. 1976 J.Fluid Mech. 73241

[13] Diamond P.H. et al. 2005 Plasma Phys. Control. Fusion 47 R35

[14] Shukla P.K. et al. 1984 Physics Reports 105227

[15] Horton W. 1990 Phys. Reports 1921

[16] Shukla P.K. et al. 1981 Phys. Rev. A 23321

[17] Diamond P.H. and McDevitt C.J. 2006 Phys.Plasmas 13032302

[18] Shukla P. K. et al. 1985 Phys. Lett. A 107461

[19] Shukla P.K. and Stenflo L. 2002 Eur. Phys. J. D 20, 103

[20] Shapiro V.D. et al. 1993 Plasma Phys. Control. Fusion 351033

[21] Pakyari A. and Pavlenko V.P. 1996 Phys. Plasmas 33661

[22] Weiland J. et al. 1981 Phys. Fluids 2493

[23] Dastgeer S. et al. 2002 Phys. Plasmas 91565

[24] Rosenbluth M.N. and Shapiro V.D. 1994 Phys. Plasmas 1222

[25] Molenaar D. et al. 2004 Physics D 196329

[26] Nezlin M.V. et al. 1996 Chaos 6309

[27] Nezlin M.V. 1994 Chaos 4109

[28] Farid T. et al. 2000 Phys. Plasmas 7166

[29] Schubert W.H. et al. 1999 J. Atmos. Sci. 561197

[30] Kossin J. P. and Schubert W.H. 2001 J. Atmos. Sci. 582196

[31] Lovelace R.V.E. 1999 ApJ 513805 
[32] Colgate S.A. and Buchler J.R. 2000 Ann. NY Acad. Sci. 898105

[33] Zhu P. et al. 1999 Phys. Plasmas 62503

[34] Morris R.C. et al. 1996 Phys. Plasmas 34513

[35] Burrell K.H et al. 1996 Plasma Phys. Control. Fusion 381313

[36] Oliver, R. 2008 Numerical prediction of primary and secondary flows in a Ranque-Hilsch vortex tube, Masters, Paper 29, http://arrow.dit.ie/engmas/29

[37] Hinton F.L. and Robertson J.A. 1984 Phys.Fluids 271243

[38] Ott E. 1972 Phys. Rev. Letters 291429

[39] Trubnikov B.A. and Zhdanov S.K. 1987 Physics reports 155137

[40] Crowdy D. 2010 Theor. Comput. Fluid Dyn. 249

[41] McCarthy D. R. et al. 1993 Phys. Fluids B 51188

[42] Dong J.Q. et al. 1998 Phys. Plasmas 54328

[43] Su X.N. et al. 1994 Phys. Plasmas 11905

[44] Drazin P.G. and Reid W.H. 2004 Hydrodynamic Stability Cambridge University Press, Cambridge, UK

[45] Spineanu F. and Vlad M. 2002 Phys. Plasmas 95125

\section{Figure 1.}

Figure caption: The system of reference and the geometry of the perturbation. The smooth surface represents the equilibrium pressure profile on which the temperature perturbation of the hot streamer is superposed. The curved arrow indicates the direction of the baroclinic velocity acceleration. The two arrows at the right side of the perturbation represents the gradients: the arrow directed to the left is $\nabla T$ of the hot streamer; the arrow directed to the negative $x$ axis is $\nabla n_{0}$ of the equilibrium density. 


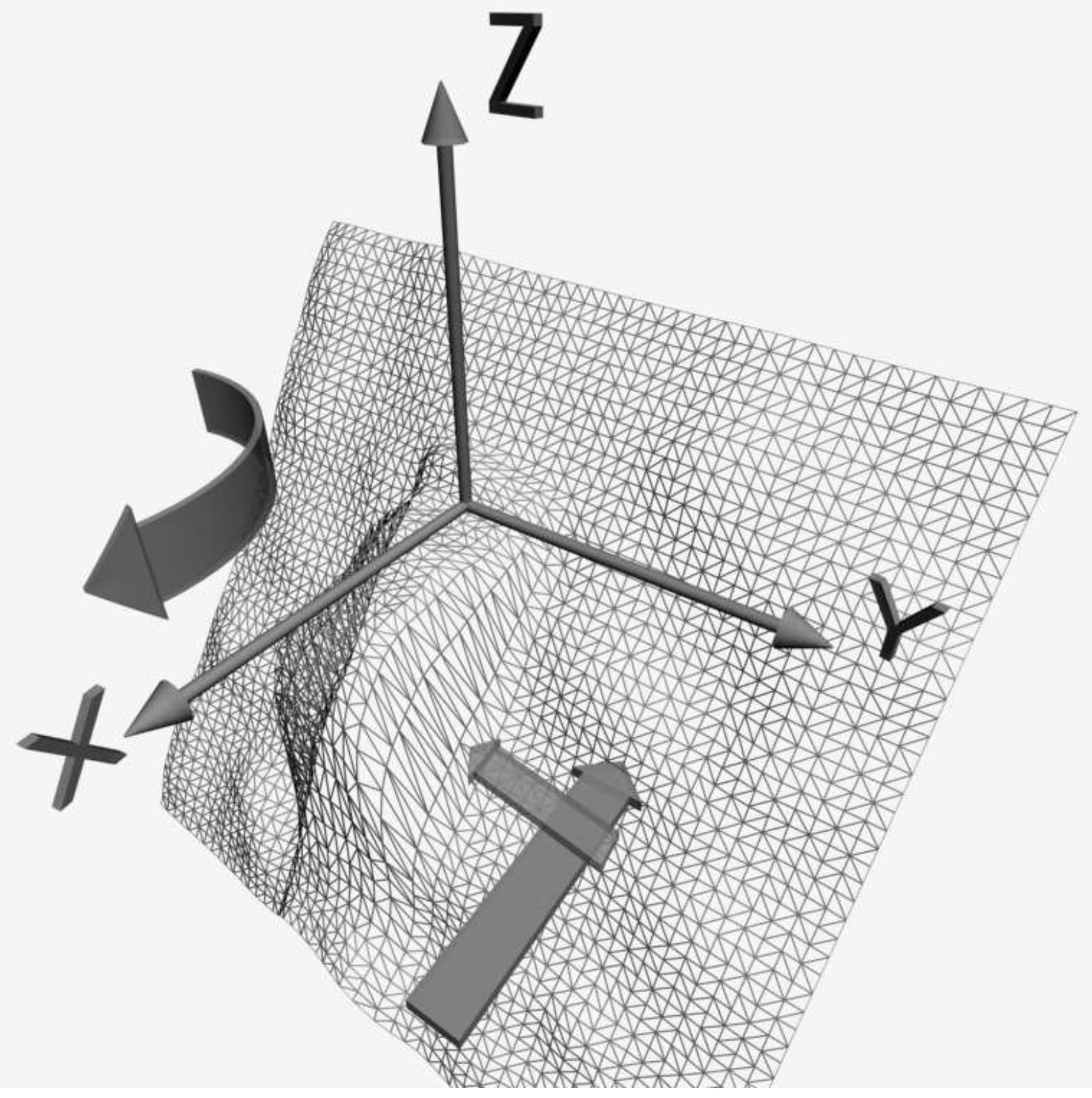

